\title{
Evaluation of relationship between air pollutant concentration and meteorological elements in winter months
}

\section{Andrzej ŻYROMSKI ${ }^{1)}$ ABCDEF, Małgorzata BINIAK-PIERÓG ${ }^{\text {1) ABCDEF, }}$ Ewa BURSZTA-ADAMIAK ${ }^{2) ~ A B C D E F}$, Zenon ZAMIAR ${ }^{1) \text { B }}$}

\author{
1) Wrocław University of Environmental and Life Sciences, Institute of Environmental Development and Protection, \\ pl. Grunwaldzki 24, 50-363 Wrocław, Poland; e-mail: andrzej.zyromski@up.wroc.pl \\ ${ }^{2)}$ Wrocław University of Environmental and Life Sciences, Institute of Environmental Engineering, pl. Grunwaldzki 24, \\ 50-363 Wrocław, Poland
}

For citation: Ż̇yromski A., Biniak-Pieróg M., Burszta-Adamiak E., Zamiar Z. 2014. Evaluation of relationship between air pollutant concentration and meteorological elements in winter months. Journal of Water and Land Development. No. 22 p. $25-32$.

\begin{abstract}
The paper presents the evaluation of the relation between meteorological elements and air pollutants' concentrations. The analysis includes daily concentrations of pollutants and variation of meteorological elements such as wind speed, air temperature and relative humidity, precipitation and total radiation at four monitoring stations located in the province of Lower Silesia in individual months of the winter half-year (November-April, according to hydrological year classification) of 2005-2009. Data on air quality and meteorological elements came from the results of research conducted in the automatic net of air pollution monitoring conducted in the range of the State Environment Monitoring. The effect of meteorological elements on analysed pollutant concentration was determined using the correlation and regression analysis at significance level $\alpha<0.05$. The occurrence of maximum concentration of $\mathrm{NO}, \mathrm{NO}_{2}, \mathrm{NO}_{\mathrm{X}}, \mathrm{SO}_{2}$ and $\mathrm{PM} 10$ occurred in the coldest months during winter season (January, February and December) confirmed the strong influence of "low emission" on air quality. Among the meteorological factors assessed wind speed was most often selected component in step wise regression procedure, then air temperature, less air relative humidity and solar radiation. In the case of a larger number of variables describing the pollution in the atmosphere, in all analyzed winter seasons the most common set of meteorological elements were wind speed and air temperature.
\end{abstract}

Key words: atmospheric pollutants, meteorological parameters, step-wise regression, winter season

\section{INTRODUCTION}

The quality of land and aquatic environment depends i.e. on the amount and type of pollutants produced in a given area [TWARDY et al. 2005]. From among many atmospheric pollutants the most harmful appear to be sulphur and nitrogen oxides that all cause water and soil degradation [SAPEK et al. 2003].

In winter time in most regions of Poland the concentration of gas and dust pollutants increases several times compared with summer time. One of the reason of this phenomenon is a considerable emission of large quantities of harmful substances during combustion processes held for heating houses and industrial buildings. Heat production in winter time, especially when using coal combustion furnaces, contributes to large concentration of mainly suspended particulate matter PM10, carbon dioxide and sulfur dioxide. Winter time signifies also more stable atmosphere and thus lesser convection and mixing of the air with consequent accumulation of pollutants at their emission sites [GLIŃSKI et al. 2000; NIDZGORSKA-LENCEWICZ, 
CzArneCKA 2014]. Moreover, in Poland there is a lack or scarcity of growing season in winter time that could absorb pollutants from the atmosphere.

The concentration of dust and gaseous pollutants is significantly affected by the meteorological conditions, such as wind, air temperature and humidity, precipitation and solar radiation. In the literature one can find numerous studies on the effect of meteorological parameters on the concentration of pollutants, $\mathrm{SO}_{2}$ and TSP - total suspended particulate [AKPINAR et al. 2009; AKPINAR, ÖZTOP 2008; BANERJEE et al. 2011; Cuhadaroglu, Demirci 1997; ÇELIK, KADI 2007; LUVSAN et al. 2012; TURALIOĞLU et al. 2005], $\mathrm{CO}, \mathrm{O}_{3}$ and $\mathrm{NO}_{\mathrm{x}}$ [KATSOULIS 1996] among others, although relations between them are not unambiguous. Increased wind speed results, in general, in decreased concentration of $\mathrm{SO}_{2}, \mathrm{NO}_{2}$ and PM10 [CZARNECKA, NIDZGORSKA-LENCEWICZ 2008; GIRI et al. 2008; PEARCE et al. 2011]. BRIDGMAN et al. [2002] found a relationship between $\mathrm{SO}_{2}$ and six meteorological parameters. The studies have shown that $\mathrm{SO}_{2}$ concentration is strongly correlated with temperature, relative humidity and wind speed. Concentration of $\mathrm{SO}_{2}$ decreased with increased humidity and decreased temperature and wind speed. Statistically significant influence on particulate matter PM10 has the sum of precipitation and their frequency expressed as the number of days with precipitation over $0.5 \mathrm{~mm}$, and also relative humidity. A lesser role is, however, played by air temperature and wind speed [CZARNECKA et al. 2007; CZARNECKA, KALBARCZYK 2008]. CAMALIER et al. [2007] have shown that $\mathrm{NO}_{\mathrm{x}}$ concentration increases with increasing air temperature and decreasing relative humidity.

The divergences in the relations between air pollution and meteorological elements result from different meteorological conditions that occur in the areas analysed and from the local topography of terrain. Therefore it seems proper to do such type of analyses for conditions that are characteristic to a given region.

The aim of the article is to evaluate the relation between meteorological elements and pollutants' concentrations, assayed at four monitoring stations localized in the Lower Silesia province. The investigations were conducted for the winter half-year (NovemberApril, according to hydrological year classification) of 2005-2009. The confirmation of the reliability of obtained relationship is the fact, that the input data of both, meteorological and pollution elements originates from the same measurement sites. Statistical analyses were done in order to determine the relations between selected air pollutants and wind speed $v$, relative air humidity $f$, total solar radiation $T$, amount of precipitation $P$ and air temperature $t p$ in individual months of the winter seasons analysed.

\section{MATERIALS AND METHODS}

Data on air quality and meteorological elements, the basis of the analyses conducted, came from the results of research conducted in the automatic net of air pollution monitoring conducted in the realm of the State Environment Monitoring. The data were provided by the Provincial Inspectorate of Environmental Protection in Wrocław. The analysis includes daily concentrations of pollutants $\left(\mathrm{NO}, \mathrm{NO}_{2}, \mathrm{NO}_{\mathrm{x}}, \mathrm{SO}_{2}\right.$, PM10, $\mathrm{CO} \mathrm{i} \mathrm{O}_{3}$ - depending on their availability at particular monitoring station) and variation of meteorological elements (precipitation, air temperature, air relative humidity, total solar radiation and wind speed) in individual months of the winter half-year (November-April) of 2005-2009.

Completeness of the data available for the years analyzed was the reason for choosing the particular monitoring stations for the present study. Four monitoring stations were chosen, localized in the Lower Silesia province: town of Dzierżoniów - ul. Piłsudskiego, Jelenia Góra-Cieplice - Park Zdrojowy, Legnica - al. Rzeczypospolitej and Wrocław-Swojec ul. Bartnicza. Their locations are shown in Fig. 1.

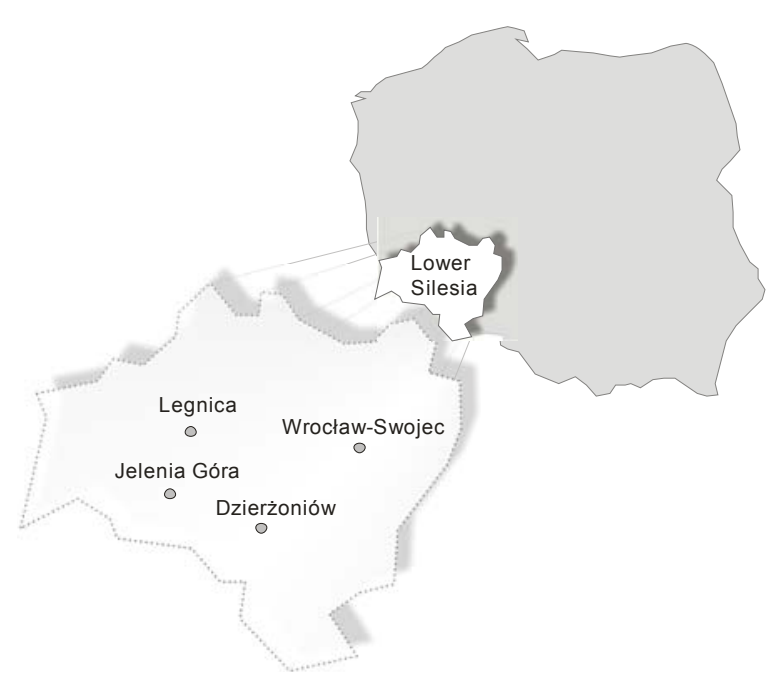

Fig. 1. Location of monitoring stations; source: own study

Stations in the towns Dzierżoniów, Legnica and Jelenia Góra are located in a town area, i.e. living quarters with commerce and services, installed to monitor air pollution in cities. The Station Wrocław-Swojec is located in a suburban area (housing and agriculture) aiming mainly at estimating the ozone air pollution in the Wrocław urban agglomeration. In Dzierżoniów in the last few years the environmentally harmful industrial plant have been shut, and the main source of air pollution remains car transportation and low emission. An effect on contamination can have the industrial plant at Bielawa - Lincoln Electric Bester - producer of half-products for LG, Samsung and Dell. In Jelenia Góra the dominant source of regional air pollution are the plants: PF „Jelfa” S.A., PMPoland S.A., JZO Sp. Ltd., Fabryka Papieru Ltd., DSE DRAEXLMAIER Ltd., DOLFAMEX Ltd., SP ZORKA, JPC „Anilux” S.A., heat generating plants and motor transport. In Legnica the copper industry plants are dominant. These are, among others, the 
Legnica Copper Mill, the „Patelec Elpena” Warp Conduit Plant and „Zanam - Legmet” plant joint with „Załam” Polkowice. At variance with the remaining stations the monitoring point at Wrocław - Swojec localized in the north-east part of the city is separated from the Wrocław proper by a complex of parks and stadiums, as well as by an Odra canal, meadows and fields. In its vicinity there are Volvo Poland Ltd plant, Chemitex (now 3M Poland Ltd) and a fuel base of a petroleum products distributor.

The analysis of the results have been divided into two stages. In the first stage we created correlation matrices, which allowed for determination of correlation coefficients $R$ between the pollution parameters analyzed and selected meteorological elements. The next stage of analysis was to obtain an optimal set of independent variables that describe best the selected air pollution parameters at the monitoring stations. To this aim the step-wise reverse regression was applied at significance level $\alpha<0.05$, using the Statistica 10 program. It assumes successive elimination from the model built on the basis of all the potential variables of those variables which have the least effect on the dependent variable, until "the best" model is reached [STANISZ 2007]. The assessment was made on the basis of corrected determination coefficients $R_{p}{ }^{2}$, allowing for the fact that $R_{p}{ }^{2}$ is calculated from a definite sample, and not from the entire population and is always smaller than the determination coefficients $R^{2}$.

\section{RESULTS AND DISCUSSION}

Tables 1-4 show compilations of statistical parameters of the data analysed for winter periods of 2005/2006-2008/2009 from individual monitoring stations.

Table 1. Statistical parameters of the daily data analysed from winter periods of 2005/2006-2008/2009 from Dzierżoniów station

\begin{tabular}{|c|c|c|c|c|c|c|c|c|c|c|c|}
\hline \multirow{2}{*}{ Month } & Statistical & $\mathrm{NO}$ & $\mathrm{NO}_{2}$ & $\mathrm{NO}_{\mathrm{x}}$ & $\mathrm{SO}_{2}$ & PM10 & \multirow{2}{*}{$\begin{array}{c}P \\
\mathrm{~mm}\end{array}$} & \multirow{2}{*}{$\begin{array}{l}t p \\
{ }^{\circ} \mathrm{C}\end{array}$} & \multirow{2}{*}{$\begin{array}{l}f \\
\%\end{array}$} & \multirow{2}{*}{$\begin{array}{c}T \\
\mathrm{~W} \cdot \mathrm{m}^{-2}\end{array}$} & \multirow{2}{*}{$\begin{array}{c}v \\
\mathrm{~m} \cdot \mathrm{s}^{-1}\end{array}$} \\
\hline & parameter & \multicolumn{5}{|c|}{$\mu \mathrm{g} \cdot \mathrm{m}^{-3}$} & & & & & \\
\hline \multirow{3}{*}{ XI } & $\min$ & 0.0 & 3.0 & 4.0 & 4.0 & 6.0 & 0.0 & -4.8 & 38.0 & 5.0 & 0.1 \\
\hline & $\max$ & 98.0 & 40.0 & 181.0 & 44.0 & 128.0 & 18.6 & 11.9 & 99.0 & 73.0 & 3.3 \\
\hline & median & 3.0 & 17.5 & 23.0 & 12.0 & 27.0 & 0.0 & 4.2 & 80.0 & 30.0 & 1.2 \\
\hline \multirow{3}{*}{ XII } & $\min$ & 0.0 & 4.0 & 5.0 & 4.0 & 8.0 & 0.0 & -9.0 & 46.0 & 2.0 & 0.1 \\
\hline & $\max$ & 157.0 & 49.0 & 286.0 & 56.0 & 249.0 & 20.0 & 8.8 & 99.0 & 41.0 & 3.0 \\
\hline & median & 3.0 & 18.0 & 22.0 & 15.0 & 28.0 & 0.0 & 0.4 & 83.0 & 15.5 & 1.2 \\
\hline \multirow{3}{*}{ I } & $\min$ & 0.0 & 4.0 & 6.0 & 4.0 & 7.0 & 0.0 & -20.4 & 44.0 & 3.0 & 0.1 \\
\hline & $\max$ & 114.0 & 76.0 & 242.0 & 120.0 & 309.0 & 15.0 & 9.3 & 99.0 & 58.0 & 4.7 \\
\hline & median & 4.0 & 20.0 & 26.5 & 20.0 & 40.5 & 0.0 & -1.7 & 82.0 & 24.0 & 1.2 \\
\hline \multirow{3}{*}{ II } & $\min$ & 0.0 & 5.0 & 6.0 & 3.0 & 8.0 & 0.0 & -12.4 & 44.0 & 5.0 & 0.1 \\
\hline & $\max$ & 56.0 & 52.0 & 132.0 & 100.0 & 181.0 & 23.4 & 9.3 & 99.0 & 106.0 & 2.9 \\
\hline & median & 4.0 & 18.0 & 23.5 & 16.0 & 31.0 & 0.2 & -0.6 & 80.5 & 46.0 & 1.2 \\
\hline \multirow{3}{*}{ III } & $\min$ & 0.0 & 2.0 & 3.0 & 3.0 & 4.0 & 0.0 & -7.0 & 47.0 & 6.0 & 0.1 \\
\hline & $\max$ & 28.0 & 37.0 & 75.0 & 45.0 & 169.0 & 23.0 & 11.2 & 99.0 & 184.0 & 3.8 \\
\hline & median & 2.0 & 18.0 & 21.0 & 12.0 & 27.0 & 0.2 & 1.9 & 76.0 & 70.0 & 1.1 \\
\hline \multirow{3}{*}{ IV } & $\min$ & 0.0 & 4.0 & 5.0 & 3.0 & 12.0 & 0.0 & 0.9 & 33.0 & 13.0 & 0.1 \\
\hline & $\max$ & 21.0 & 37.0 & 65.0 & 23.0 & 97.0 & 36.9 & 14.0 & 99.0 & 243.0 & 2.7 \\
\hline & median & 1.0 & 7.0 & 20.0 & 8.0 & 29.0 & 0.0 & 6.5 & 62.5 & 136.0 & 1.0 \\
\hline
\end{tabular}

Explanations: $P$ - precipitation, $t p$ - air temperature, $f$ - air relative humidity, $T$ - total solar radiation, $v$ - wind speed. Source: own study.

Table 2. Statistical parameters of the daily data analyzed from winter periods of 2005/2006-2008/2009 from Legnica station

\begin{tabular}{|c|c|c|c|c|c|c|c|c|c|c|}
\hline \multirow{2}{*}{ Month } & \multirow{2}{*}{$\begin{array}{l}\text { Statistical } \\
\text { parameter }\end{array}$} & $\mathrm{NO}$ & $\mathrm{NO}_{2}$ & $\mathrm{NO}_{\mathrm{x}}$ & $\mathrm{SO}_{2}$ & \multirow{2}{*}{$\begin{array}{c}P \\
\mathrm{~mm}\end{array}$} & \multirow{2}{*}{$\begin{array}{l}t p \\
{ }^{\circ} \mathrm{C}\end{array}$} & \multirow{2}{*}{$\begin{array}{l}f \\
\%\end{array}$} & \multirow{2}{*}{$\begin{array}{c}T \\
\mathrm{~W} \cdot \mathrm{m}^{-2}\end{array}$} & \multirow{2}{*}{$\begin{array}{c}v \\
\mathrm{~m} \cdot \mathrm{s}^{-1}\end{array}$} \\
\hline & & \multicolumn{4}{|c|}{$\mu \mathrm{g} \cdot \mathrm{m}^{-3}$} & & & & & \\
\hline 1 & 2 & 3 & 4 & 5 & 6 & 7 & 8 & 9 & 10 & 11 \\
\hline \multirow{3}{*}{ XI } & $\min$ & 1.0 & 8.0 & 10.0 & 5.0 & 0.0 & -4.3 & 49.0 & 4.0 & 0.0 \\
\hline & $\max$ & 99.0 & 46.0 & 196.0 & 40.0 & 19.0 & 11.5 & 99.0 & 75.0 & 3.0 \\
\hline & median & 9.0 & 25.0 & 39.0 & 11.0 & 0.2 & 4.8 & 82.0 & 30.0 & 1.1 \\
\hline \multirow{3}{*}{ XII } & $\min$ & 0.0 & 9.0 & 10.0 & 5.0 & 0.0 & -6.9 & 64.0 & 1.0 & 0.1 \\
\hline & $\max$ & 100.0 & 56.0 & 208.0 & 47.0 & 16.3 & 8.9 & 99.0 & 47.0 & 3.3 \\
\hline & median & 6.0 & 27.0 & 35.5 & 16.0 & 0.1 & 1.6 & 84.0 & 14.0 & 1.2 \\
\hline \multirow{3}{*}{ I } & $\min$ & 0.0 & 6.0 & 7.0 & 4.0 & 0.0 & -19.4 & 58.0 & 3.0 & 0.2 \\
\hline & $\max$ & 196.0 & 104.0 & 405.0 & 125.0 & 13.4 & 11.5 & 99.0 & 83.0 & 3.9 \\
\hline & median & 6.0 & 28.0 & 39.0 & 18.0 & 0.2 & -0.3 & 82.0 & 23.0 & 1.3 \\
\hline \multirow{3}{*}{ II } & $\min$ & 0.0 & 11.0 & 12.0 & 6.0 & 0.0 & -12.3 & 43.0 & 8.0 & 0.2 \\
\hline & $\max$ & 83.0 & 53.0 & 177.0 & 72.0 & 12.0 & 10.5 & 99.0 & 118.0 & 3.4 \\
\hline & median & 5.5 & 27.5 & 36.5 & 16.0 & 0.2 & 1.1 & 80.0 & 46.5 & 1.1 \\
\hline
\end{tabular}


cont. tab. 2

\begin{tabular}{|c|l|r|r|r|r|r|r|r|r|r|}
\hline 1 & \multicolumn{1}{|c|}{2} & \multicolumn{1}{c|}{3} & \multicolumn{1}{c|}{4} & \multicolumn{1}{c|}{5} & \multicolumn{1}{c|}{6} & \multicolumn{1}{c|}{7} & \multicolumn{1}{c|}{8} & 9 & 10 & 11 \\
\hline \multirow{3}{*}{ III } & min & 0.0 & 7.0 & 8.0 & 3.0 & 0.0 & -4.8 & 53.0 & 11.0 & 0.0 \\
& max & 75.0 & 46.0 & 161.0 & 34.0 & 14.2 & 11.8 & 99.0 & 234.0 & 3.2 \\
& median & 4.0 & 23.0 & 28.0 & 12.0 & 0.0 & 4.1 & 73.0 & 89.5 & 1.2 \\
\hline \multirow{3}{*}{ IV } & min & 0.0 & 9.0 & 9.0 & 2.0 & 0.0 & 2.0 & 34.0 & 18.0 & 0.1 \\
& max & 63.0 & 55.0 & 151.0 & 20.0 & 14.2 & 18.4 & 99.0 & 285.0 & 2.5 \\
& median & 3.0 & 23.0 & 28.0 & 7.0 & 0.0 & 9.1 & 61.0 & 179.0 & 1.0 \\
\hline
\end{tabular}

Explanations as in Tab. 1.

Source: own study.

Table 3. Statistical parameters of the daily data analysed from winter periods of 2005/2006-2008/2009 from Wrocław Swojec station

\begin{tabular}{|c|c|c|c|c|c|c|c|c|c|c|}
\hline \multirow{2}{*}{ Month } & Statistical & $\mathrm{NO}$ & $\mathrm{NO}_{2}$ & $\mathrm{NO}_{\mathrm{x}}$ & $\mathrm{O}_{3}$ & \multirow{2}{*}{$\begin{array}{c}P \\
\mathrm{~mm}\end{array}$} & \multirow{2}{*}{$\begin{array}{l}t p \\
{ }^{t} \mathrm{C}\end{array}$} & \multirow{2}{*}{$\begin{array}{l}f \\
\%\end{array}$} & \multirow{2}{*}{$\begin{array}{c}T \\
\mathrm{~W} \cdot \mathrm{m}^{-2}\end{array}$} & \multirow{2}{*}{$\begin{array}{c}v \\
\mathrm{~m} \cdot \mathrm{s}^{-1}\end{array}$} \\
\hline & parameter & \multicolumn{4}{|c|}{$\mu \mathrm{g} \cdot \mathrm{m}^{-3}$} & & & & & \\
\hline \multirow{3}{*}{ XI } & $\min$ & 0.0 & 5.0 & 5.0 & 4.0 & 0.0 & -3.9 & 64.0 & 8.3 & 0.0 \\
\hline & $\max$ & 33.0 & 36.0 & 78.0 & 56.0 & 11.4 & 9.2 & 99.0 & 92.1 & 6.1 \\
\hline & median & 2.5 & 17.5 & 23.0 & 24.0 & 0.2 & 2.4 & 87.5 & 30.7 & 2.1 \\
\hline \multirow{3}{*}{ XII } & $\min$ & 0.0 & 5.0 & 5.0 & 4.0 & 0.0 & -7.8 & 73.0 & 3.0 & 0.0 \\
\hline & $\max$ & 54.0 & 39.0 & 122.0 & 56.0 & 30.7 & 9.3 & 99.0 & 45.0 & 5.5 \\
\hline & median & 1.0 & 19.0 & 21.0 & 24.0 & 0.2 & 1.4 & 87.0 & 17.5 & 1.8 \\
\hline \multirow{3}{*}{ I } & $\min$ & 0.0 & 2.0 & 2.0 & 5.0 & 0.0 & $\begin{array}{l}-19.9 \\
\end{array}$ & 61.0 & 7.0 & 0.2 \\
\hline & $\max$ & 30.0 & 75.0 & 104.0 & 71.0 & 43.6 & 10.3 & 98.0 & 84.0 & 7.8 \\
\hline & median & 1.0 & 20.0 & 21.0 & 36.5 & 0.2 & 0.6 & 84.0 & 27.0 & 2.5 \\
\hline \multirow{3}{*}{ II } & $\min$ & 0.0 & 5.0 & 5.0 & 5.0 & 0.0 & -12.4 & 48.0 & 10.0 & 0.2 \\
\hline & $\max$ & 48.0 & 48.0 & 116.0 & 73.0 & 8.8 & 9.4 & 98.0 & 121.0 & 5.2 \\
\hline & median & 1.0 & 22.0 & 25.0 & 38.0 & 0.0 & -0.1 & 87.0 & 45.0 & 2.6 \\
\hline \multirow{3}{*}{ III } & $\min$ & 0.0 & 4.0 & 4.0 & 23.0 & 0.0 & -5.4 & 49.0 & 11.0 & 0.2 \\
\hline & $\max$ & 7.0 & 37.0 & 43.0 & 103.0 & 10.0 & 10.2 & 98.0 & 189.0 & 6.7 \\
\hline & median & 1.0 & 16.0 & 18.0 & 61.0 & 0.0 & 3.3 & 81.0 & 88.0 & 2.0 \\
\hline \multirow{3}{*}{ IV } & $\min$ & 0.0 & 3.0 & 3.0 & 41.0 & 0.0 & 2.6 & 37.0 & 22.0 & 0.2 \\
\hline & $\max$ & 15.0 & 33.0 & 55.0 & 101.0 & 18.8 & 17.2 & 98.0 & 257.0 & 4.7 \\
\hline & median & 1.0 & 14.0 & 15.0 & 69.0 & 0.0 & 8.8 & 67.0 & 179.0 & 1.8 \\
\hline
\end{tabular}

Explanations as in Tab. 1.

Source: own study.

Table 4. Statistical parameters of the daily data analyzed from winter periods of 2005/2006-2008/2009 from Jelenia Góra station

\begin{tabular}{|c|c|c|c|c|c|c|c|c|c|c|c|c|c|}
\hline \multirow{2}{*}{ Month } & \multirow{2}{*}{$\begin{array}{l}\text { Statistical } \\
\text { parameter }\end{array}$} & $\mathrm{NO}$ & $\mathrm{NO}_{2}$ & $\mathrm{NO}_{\mathrm{x}}$ & $\mathrm{SO}_{2}$ & PM10 & $\mathrm{CO}$ & $\mathrm{O}_{3}$ & \multirow{2}{*}{$\begin{array}{c}P \\
\mathrm{~mm}\end{array}$} & \multirow{2}{*}{$\begin{array}{l}t p \\
{ }^{\circ} \mathrm{C}\end{array}$} & \multirow{2}{*}{$\begin{array}{l}f \\
\%\end{array}$} & \multirow{2}{*}{$\begin{array}{c}T \\
\mathrm{~W} \cdot \mathrm{m}^{-2}\end{array}$} & \multirow{2}{*}{$\begin{array}{c}v \\
\mathrm{~m} \cdot \mathrm{s}^{-1}\end{array}$} \\
\hline & & \multicolumn{7}{|c|}{$\mu \mathrm{g} \cdot \mathrm{m}^{-3}$} & & & & & \\
\hline \multirow{3}{*}{ XI } & $\min$ & 0.0 & 5.0 & 5.0 & 2.0 & 6.0 & 118.0 & 2.0 & 0.0 & -7.0 & 41.0 & 2.0 & 0.0 \\
\hline & $\max$ & 73.0 & 45.0 & 145.0 & 41.0 & 219.0 & 2584.0 & 62.0 & 126.1 & 8.4 & 98.0 & 58.0 & 2.0 \\
\hline & median & 7.0 & 21.0 & 32.0 & 10.0 & 42.0 & 623.0 & 24.0 & 0.2 & 0.5 & 82.0 & 25.0 & 0.4 \\
\hline \multirow{3}{*}{ XII } & $\min$ & 0.0 & 6.0 & 6.0 & 3.0 & 4.0 & 181.0 & 1.0 & 0.0 & -11.0 & 54.0 & 3.0 & 0.0 \\
\hline & $\max$ & 74.0 & 58.0 & 158.0 & 54.0 & 387.0 & 3625.0 & 62.0 & 16.8 & 5.2 & 98.0 & 34.0 & 1.8 \\
\hline & median & 5.0 & 22.0 & 30.0 & 13.0 & 33.0 & 558.0 & 26.0 & 0.2 & -1.3 & 84.0 & 14.0 & 0.4 \\
\hline \multirow{3}{*}{ I } & $\min$ & 0.0 & 8.0 & 10.0 & 4.0 & 5.0 & 221.0 & 1.0 & 0.0 & -15.9 & 58.0 & 1.0 & 0.0 \\
\hline & $\max$ & 65.0 & 78.0 & 172.0 & 89.0 & 374.0 & 4063.0 & 64.0 & 20.6 & 7.3 & 98.0 & 50.0 & 2.3 \\
\hline & median & 8.0 & 27.0 & 37.0 & 19.0 & 56.0 & 703.0 & 23.0 & 0.0 & -2.3 & 80.0 & 18.0 & 0.3 \\
\hline \multirow{3}{*}{ II } & $\min$ & 0.0 & 7.0 & 8.0 & 1.0 & 9.0 & 223.0 & 7.0 & 0.0 & -15.1 & 42.0 & 3.0 & 0.0 \\
\hline & $\max$ & 35.0 & 60.0 & 107.0 & 53.0 & 404.0 & 2390.0 & 78.0 & 31.5 & 7.9 & 98.0 & 115.0 & 1.7 \\
\hline & median & 3.0 & 20.0 & 25.0 & 13.0 & 41.0 & 577.0 & 38.0 & 0.0 & -1.0 & 77.0 & 43.0 & 0.6 \\
\hline \multirow{3}{*}{ III } & $\min$ & 0.0 & 7.0 & 8.0 & 3.0 & 7.0 & 149.0 & 26.0 & 0.0 & -6.5 & 47.0 & 8.0 & 0.0 \\
\hline & $\max$ & 17.0 & 36.0 & 60.0 & 30.0 & 153.0 & 1246.0 & 103.0 & 15.4 & 9.0 & 97.0 & 195.0 & 2.5 \\
\hline & median & 3.0 & 20.0 & 24.0 & 10.0 & 50.0 & 614.0 & 56.0 & 0.0 & 1.4 & 72.0 & 79.0 & 0.5 \\
\hline \multirow{3}{*}{ IV } & $\min$ & 0.0 & 5.0 & 5.0 & 2.0 & 13.0 & 136.0 & 44.0 & 0.0 & -1.0 & 30.0 & 19.0 & 0.0 \\
\hline & $\max$ & 11.0 & 30.0 & 41.0 & 14.0 & 89.0 & 950.0 & 118.0 & 16.8 & 13.7 & 90.0 & 257.0 & 1.4 \\
\hline & median & 3.0 & 17.0 & 21.0 & 6.0 & 36.0 & 373.0 & 65.0 & 0.0 & 5.7 & 63.0 & 167.0 & 0.5 \\
\hline
\end{tabular}

Explanations as in Tab. 1.

Source: own study. 
At Legnica station the highest concentration of all the parameters analyzed took place in January. Similar regularity was not observed with the remaining stations. The highest values of NO concentration were observed in December at Dzierżoniów $\left(157 \mu \mathrm{g} \cdot \mathrm{m}^{-3}\right)$, Jelenia Góra $\left(74.0 \mu \mathrm{g} \cdot \mathrm{m}^{-3}\right)$ and Wrocław - Swojec $\left(54.0 \mu \mathrm{g} \cdot \mathrm{m}^{-3}\right)$. Maximal $\mathrm{NO}_{2}$ concentrations occurred in January at Jelenia Góra $\left(78.0 \mu \mathrm{g} \cdot \mathrm{m}^{-3}\right)$, Dzierżoniów $\left(76.0 \mu \mathrm{g} \cdot \mathrm{m}^{-3}\right)$ and Wrocław - Swojec $\left(75.0 \mu \mathrm{g} \cdot \mathrm{m}^{-3}\right)$. stations. The highest concentration of $\mathrm{NO}_{\mathrm{x}}$ was in December at Dzierżoniów and Wrocław - Swojec $\left(75.0 \mathrm{i} 186.0 \mu \mathrm{g} \cdot \mathrm{m}^{-3}\right)$, and in January at Jelenia Góra $\left(172.0 \mu \mathrm{g} \cdot \mathrm{m}^{-3}\right)$. Maximal concentration of $\mathrm{O}_{3}(103.0$ $\left.\mu \mathrm{g} \cdot \mathrm{m}^{-3}\right)$ was in March at Jelenia Góra and Wrocław Swojec. The highest concentrations of the remaining pollutants i.e. $\mathrm{SO}_{2}, \mathrm{PM} 10$ and $\mathrm{CO}$ in most of the cases was observed in January.

a)

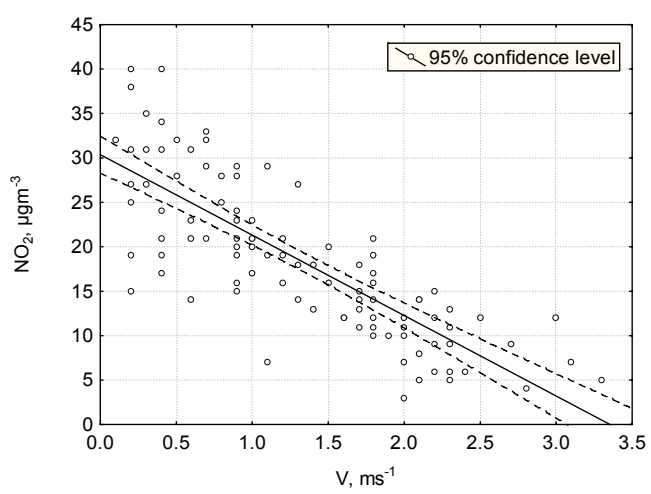

c)

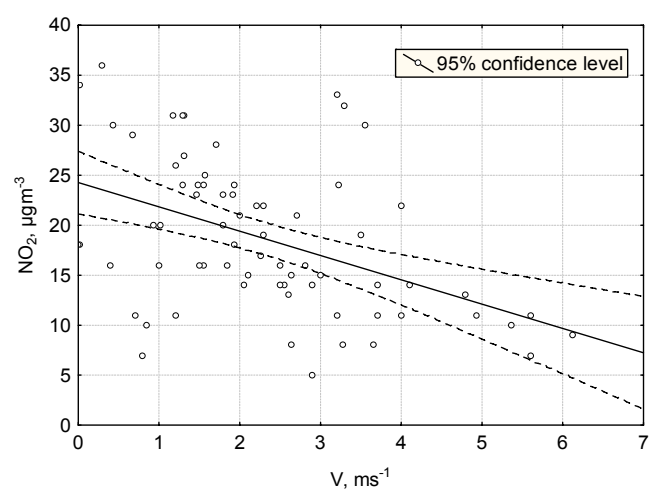

Analysis of historical material on meteorological data considered here indicate that the four winter periods analyzed exhibit a considerable differentiation of the meteorological data that can occur in the areas determined by the four basic stations.

The correlation analyses performed indicate that wind speed is the meteorological element correlated most with the concentrations analyzed independent of the month, the relation being in each case of inverse character. In most of the cases the values of the correlation coefficients obtained $\mathrm{R}$ were highest for the relation between wind speed and $\mathrm{NO}_{2}$ at Dzierżoniów, Jelenia Góra and Legnica $(-0.43<R<-0.80)$. Representative plots of scatter in measured $\mathrm{NO}_{2}$ and wind speed for November of 2005/2006-2008/2009 at individual monitoring stations are shown in Fig. 2.

b)

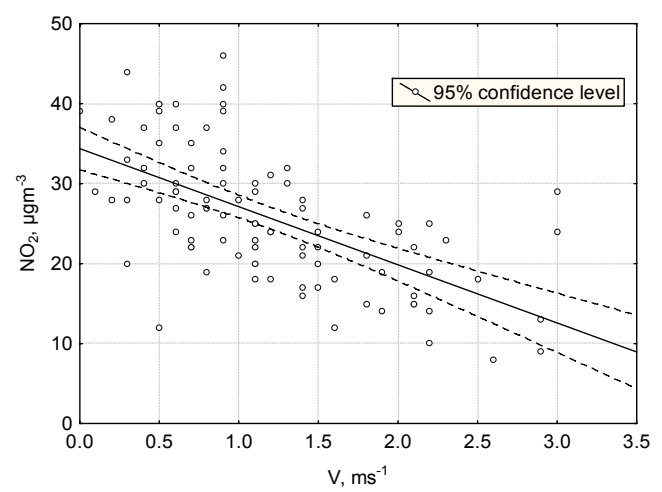

d)

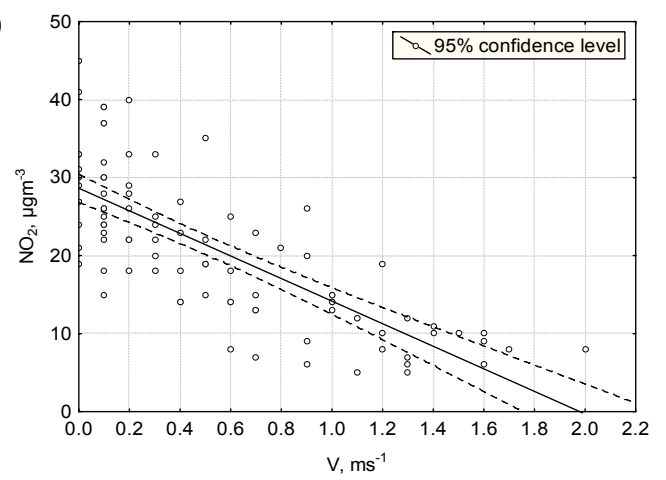

Fig. 2. Scatter in $\mathrm{NO}_{2}$ measurements and wind speed in November for years 2005-2008 at: a) Dzierżoniów $(R=-0.80)$, b) Legnica $(R=-0.67)$, c) Wrocław-Swojec $(R=-0.45)$, d) Jelenia Góra $(R=-0.78)$ monitoring stations; source: own study

A positive correlation was found between wind speed and $\mathrm{O}_{3}$ concentration at Jelenia Góra and Wrocław - Swojec stations. It was high in November, December and January, the correlation coefficient $R$ assuming the values $0.46-0.86$.

The significant values obtained of the coefficients $\mathrm{R}$ for the correlation between pollutant concentration and air temperature indicate at a reverse relation between them independently of station and type of pollution. Significant correlations were obtained for the months of December and January. However, in November this element influenced only $\mathrm{SO}_{2}$ concentration.
In the case of the sums of atmospheric precipitation no significant relations were found for most of the months of the calendar winter season (December, January, February). Only for November and April weak correlations were found at Dzierżoniów and Legnica, whereas in March at Jelenia Góra. Weak or medium correlation was testified by the values of $R$ coefficient for relations between relative humidity of the air and its quality. The concentration of pollutants increases with increasing humidity. Only in the case of $\mathrm{O}_{3}$ the correlation was negative. 
Table 5. Statistically significant parameters and values of corrected determination coefficients $R_{p}{ }^{2}$ in the years 2005/20062008/2009

\begin{tabular}{|c|c|c|c|c|c|c|c|c|}
\hline \multirow{2}{*}{ Station } & \multirow{2}{*}{ Parameter } & $\mathrm{NO}$ & $\mathrm{NO}_{2}$ & $\mathrm{NO}_{\mathrm{x}}$ & $\mathrm{SO}_{2}$ & PM10 & $\mathrm{CO}$ & $\mathrm{O}_{3}$ \\
\hline & & \multicolumn{7}{|c|}{$\mu \mathrm{g} \cdot \mathrm{m}^{-3}$} \\
\hline \multicolumn{9}{|c|}{ November of years 2005-2008 } \\
\hline Dzierżoniów & $\begin{array}{l}\text { P. I. } \\
R_{p}{ }^{2}\end{array}$ & $\begin{array}{c}v \\
0.24\end{array}$ & $\begin{array}{c}v \\
0.64\end{array}$ & $\begin{array}{c}v \\
0.38\end{array}$ & $\begin{array}{l}t p, v \\
0.48\end{array}$ & $\begin{array}{c}v \\
0.45\end{array}$ & - & $\begin{array}{l}- \\
-\end{array}$ \\
\hline Legnica & $\begin{array}{l}\text { P. I. } \\
R_{p}^{2}\end{array}$ & $\begin{array}{c}v \\
0.18 \\
\end{array}$ & $\begin{array}{c}v \\
0.37 \\
\end{array}$ & $\begin{array}{c}v \\
0.23 \\
\end{array}$ & $\begin{array}{l}t p, v \\
0.30\end{array}$ & $\begin{array}{l}- \\
- \\
\end{array}$ & $\begin{array}{c}v \\
0.26 \\
\end{array}$ & $\begin{array}{l}- \\
- \\
\end{array}$ \\
\hline Wrocław-Swojec & $\begin{array}{l}\text { P. I. } \\
R_{p}{ }^{2} \\
\end{array}$ & $\begin{array}{c}v \\
0.16 \\
\end{array}$ & $\begin{array}{c}v \\
0.19 \\
\end{array}$ & $\begin{array}{c}v \\
0.21 \\
\end{array}$ & $\begin{array}{l}- \\
- \\
\end{array}$ & $\begin{array}{l}- \\
- \\
\end{array}$ & $\begin{array}{l}- \\
- \\
\end{array}$ & $\begin{array}{l}f, v \\
0.68 \\
\end{array}$ \\
\hline Jelenia Góra & $\begin{array}{l}\text { P. I. } \\
R_{p}^{2}\end{array}$ & $\begin{array}{c}v \\
0.33 \\
\end{array}$ & $\begin{array}{c}v \\
0.59 \\
\end{array}$ & $\begin{array}{c}t p, T, v \\
0.54\end{array}$ & $\begin{array}{l}t p, v \\
0.49 \\
\end{array}$ & $\begin{array}{c}t p, T, v \\
0.52\end{array}$ & $\begin{array}{c}t p, T, v \\
0.59\end{array}$ & $\begin{array}{l}- \\
- \\
\end{array}$ \\
\hline \multicolumn{9}{|c|}{ December of years 2005-2008 } \\
\hline Dzierżoniów & $\begin{array}{l}\text { P. I. } \\
R_{p}{ }^{2} \\
\end{array}$ & $\begin{array}{c}v \\
0.20 \\
\end{array}$ & $\begin{array}{c}v \\
0.45 \\
\end{array}$ & $\begin{array}{c}v \\
0.29 \\
\end{array}$ & $\begin{array}{c}t p, T, v \\
0.60\end{array}$ & $\begin{array}{c}t p, T, v \\
0.55\end{array}$ & - & $\begin{array}{l}- \\
- \\
\end{array}$ \\
\hline Legnica & $\begin{array}{l}\text { P. I. } \\
R_{p}^{2}\end{array}$ & $\begin{array}{l}T, v \\
0.38\end{array}$ & $\begin{array}{c}f, T, v \\
0.37\end{array}$ & $\begin{array}{l}T, v \\
0.39\end{array}$ & $\begin{array}{c}t p, T, v \\
0.53\end{array}$ & $\begin{array}{l}- \\
-\end{array}$ & $\begin{array}{c}f, T, v \\
0.55\end{array}$ & $\begin{array}{l}- \\
- \\
\end{array}$ \\
\hline Wrocław-Swojec & $\begin{array}{l}\text { P. I. } \\
R_{p}^{2}\end{array}$ & $\begin{array}{c}t p \\
0.14 \\
\end{array}$ & $\begin{array}{c}v \\
0.13 \\
\end{array}$ & $\begin{array}{l}f, T \\
0.20 \\
\end{array}$ & $\begin{array}{l}- \\
- \\
\end{array}$ & $\begin{array}{l}- \\
- \\
\end{array}$ & $\begin{array}{l}- \\
- \\
\end{array}$ & $\begin{array}{l}f, v \\
0.44\end{array}$ \\
\hline Jelenia Góra & $\begin{array}{l}\text { P. I. } \\
R_{p}^{2} \\
\end{array}$ & $\begin{array}{c}t p, f, T \\
0.46 \\
\end{array}$ & $\begin{array}{c}v \\
0.48 \\
\end{array}$ & $\begin{array}{c}f, T, v \\
0.51 \\
\end{array}$ & $\begin{array}{c}t p, v, T \\
0.57 \\
\end{array}$ & $\begin{array}{c}t p, f, T \\
0.58 \\
\end{array}$ & $\begin{array}{c}t p, f, T \\
0.56 \\
\end{array}$ & $\begin{array}{l}f, v \\
0.76 \\
\end{array}$ \\
\hline \multicolumn{9}{|c|}{ January of years 2006-2009 } \\
\hline Dzierżoniów & $\begin{array}{l}\text { P. I. } \\
R_{p}{ }^{2} \\
\end{array}$ & $\begin{array}{c}v \\
0.24 \\
\end{array}$ & $\begin{array}{c}v \\
0.49 \\
\end{array}$ & $\begin{array}{c}v \\
0.33 \\
\end{array}$ & $\begin{array}{c}t p, f, v \\
0.59 \\
\end{array}$ & $\begin{array}{l}t p, v \\
0.45\end{array}$ & $\begin{array}{l}- \\
- \\
\end{array}$ & $\begin{array}{l}- \\
- \\
\end{array}$ \\
\hline Legnica & $\begin{array}{l}\text { P. I. } \\
R_{p}{ }^{2}\end{array}$ & $\begin{array}{l}t p, v \\
0.25\end{array}$ & $\begin{array}{c}t p, T, v \\
0.55\end{array}$ & $\begin{array}{l}t p, v \\
0.34\end{array}$ & $\begin{array}{c}t p, f, v \\
0.60\end{array}$ & $\begin{array}{l}- \\
- \\
\end{array}$ & $\begin{array}{l}t p, v \\
0.43\end{array}$ & $\begin{array}{l}- \\
- \\
\end{array}$ \\
\hline Wrocław-Swojec & $\begin{array}{l}\text { P. I. } \\
R_{p}{ }^{2} \\
\end{array}$ & $\begin{array}{c}v \\
0.19 \\
\end{array}$ & $\begin{array}{l}f, T \\
0.31 \\
\end{array}$ & $\begin{array}{c}v \\
0.27 \\
\end{array}$ & $\begin{array}{l}- \\
- \\
\end{array}$ & $\begin{array}{l}- \\
- \\
\end{array}$ & $\begin{array}{l}- \\
- \\
\end{array}$ & $\begin{array}{l}f, v \\
0.49 \\
\end{array}$ \\
\hline Jelenia Góra & $\begin{array}{l}\text { P. I. } \\
R_{p}^{2} \\
\end{array}$ & $\begin{array}{l}t p, v \\
0.43\end{array}$ & $\begin{array}{l}t p, v \\
0.64\end{array}$ & $\begin{array}{l}t p, v \\
0.54\end{array}$ & $\begin{array}{c}t p \\
0.62\end{array}$ & $\begin{array}{c}t p \\
0.52 \\
\end{array}$ & $\begin{array}{c}t p \\
0.52 \\
\end{array}$ & $\begin{array}{c}v \\
0.65\end{array}$ \\
\hline \multicolumn{9}{|c|}{ February of years 2006-2009 } \\
\hline Dzierżoniów & $\begin{array}{l}\text { P. I. } \\
R_{p}^{2} \\
\end{array}$ & $\begin{array}{c}v \\
0.25 \\
\end{array}$ & $\begin{array}{c}v \\
0.51 \\
\end{array}$ & $\begin{array}{c}v \\
0.38 \\
\end{array}$ & $\begin{array}{l}t p, v \\
0.45\end{array}$ & $\begin{array}{c}v \\
0.37 \\
\end{array}$ & $\begin{array}{l}- \\
-\end{array}$ & $\begin{array}{l}- \\
- \\
\end{array}$ \\
\hline Legnica & $\begin{array}{l}\text { P. I. } \\
R_{p}^{2}\end{array}$ & $\begin{array}{c}v \\
0.11 \\
\end{array}$ & $\begin{array}{c}v \\
0.18 \\
\end{array}$ & $\begin{array}{c}v \\
0.15 \\
\end{array}$ & $\begin{array}{c}t p \\
0.31 \\
\end{array}$ & $\begin{array}{l}- \\
- \\
\end{array}$ & $\begin{array}{c}v \\
0.14 \\
\end{array}$ & $\begin{array}{l}- \\
- \\
\end{array}$ \\
\hline Wrocław-Swojec & $\begin{array}{l}\text { P. I. } \\
R_{p}^{2} \\
\end{array}$ & $\begin{array}{c}v \\
0.16\end{array}$ & $\begin{array}{c}v \\
0.30\end{array}$ & $\begin{array}{c}v \\
0.30\end{array}$ & $\begin{array}{l}- \\
- \\
\end{array}$ & $\begin{array}{l}- \\
- \\
\end{array}$ & $\begin{array}{l}- \\
-\end{array}$ & $\begin{array}{l}T, v \\
0.57\end{array}$ \\
\hline Jelenia Góra & $\begin{array}{l}\text { P. I. } \\
R_{p}^{2} \\
\end{array}$ & $\begin{array}{c}v \\
0.24 \\
\end{array}$ & $\begin{array}{c}v \\
0.49 \\
\end{array}$ & $\begin{array}{c}v \\
0.40 \\
\end{array}$ & $\begin{array}{l}t p, v \\
0.50 \\
\end{array}$ & $\begin{array}{c}v \\
0.26 \\
\end{array}$ & $\begin{array}{c}v \\
0.41 \\
\end{array}$ & $\begin{array}{l}f, v \\
0.43 \\
\end{array}$ \\
\hline \multicolumn{9}{|c|}{ March of years 2006-2009 } \\
\hline Dzierżoniów & $\begin{array}{l}\text { P. I. } \\
R_{p}^{2} \\
\end{array}$ & $\begin{array}{l}T, v \\
0.38 \\
\end{array}$ & $\begin{array}{l}f, v \\
0.57 \\
\end{array}$ & $\begin{array}{l}T, v \\
0.49 \\
\end{array}$ & $\begin{array}{c}v \\
0.32 \\
\end{array}$ & $\begin{array}{c}v \\
0.21 \\
\end{array}$ & $\begin{array}{l}- \\
- \\
\end{array}$ & $\begin{array}{l}- \\
- \\
\end{array}$ \\
\hline Legnica & $\begin{array}{l}\text { P. I. } \\
R_{p}{ }^{2} \\
\end{array}$ & $\begin{array}{l}t p, v \\
0.25 \\
\end{array}$ & $\begin{array}{c}v \\
0.38 \\
\end{array}$ & $\begin{array}{c}v \\
0.25 \\
\end{array}$ & $\begin{array}{c}v \\
0.17 \\
\end{array}$ & $\begin{array}{l}- \\
- \\
\end{array}$ & $\begin{array}{c}v \\
0.37 \\
\end{array}$ & $\begin{array}{l}- \\
- \\
\end{array}$ \\
\hline Wrocław-Swojec & $\begin{array}{l}\text { P. I. } \\
R_{p}{ }^{2} \\
\end{array}$ & $\begin{array}{c}\mathrm{v} \\
0.13 \\
\end{array}$ & $\begin{array}{l}\mathrm{T}, \mathrm{v} \\
0.23\end{array}$ & $\begin{array}{c}\mathrm{v} \\
0.13 \\
\end{array}$ & $\begin{array}{l}- \\
-\end{array}$ & - & $\begin{array}{l}- \\
- \\
\end{array}$ & $\begin{array}{c}t p, T, v \\
0.36\end{array}$ \\
\hline Jelenia Góra & $\begin{array}{l}\text { P. I. } \\
R_{p}^{2} \\
\end{array}$ & $\begin{array}{l}t p, v \\
0.24\end{array}$ & $\begin{array}{c}v \\
0.59 \\
\end{array}$ & $\begin{array}{c}v \\
0.43 \\
\end{array}$ & $\begin{array}{l}t p, v \\
0.51 \\
\end{array}$ & $\begin{array}{c}v \\
0.30 \\
\end{array}$ & $\begin{array}{c}v \\
0.54 \\
\end{array}$ & $\begin{array}{l}t p, f \\
0.22 \\
\end{array}$ \\
\hline \multicolumn{9}{|c|}{ April of years 2006-2009 } \\
\hline Dzierżoniów & $\begin{array}{l}\text { P. I. } \\
R_{p}^{2} \\
\end{array}$ & $\begin{array}{c}P, T, v \\
0.43\end{array}$ & $\begin{array}{c}P, T, v \\
0.47\end{array}$ & $\begin{array}{c}P, T, v \\
0.46\end{array}$ & $\begin{array}{c}P, T, v \\
0.30\end{array}$ & $\begin{array}{l}P, T \\
0.33 \\
\end{array}$ & $\begin{array}{l}- \\
- \\
\end{array}$ & $\begin{array}{l}- \\
- \\
\end{array}$ \\
\hline Legnica & $\begin{array}{l}\text { P. I. } \\
R_{p}^{2} \\
\end{array}$ & $\begin{array}{c}v \\
0.10\end{array}$ & $\begin{array}{c}v \\
0.19\end{array}$ & $\begin{array}{c}v \\
0.15\end{array}$ & $\begin{array}{l}- \\
- \\
\end{array}$ & $\begin{array}{l}- \\
-\end{array}$ & $\begin{array}{c}v \\
0.17 \\
\end{array}$ & - \\
\hline Wrocław-Swojec & $\begin{array}{l}\text { P. I. } \\
R_{p}^{2} \\
\end{array}$ & $\begin{array}{c}\# \\
\# \# \\
\end{array}$ & $\begin{array}{c}\# \\
\# \# \\
\end{array}$ & $\begin{array}{c}\# \\
\# \# \\
\end{array}$ & $\begin{array}{l}- \\
- \\
\end{array}$ & $\begin{array}{l}- \\
- \\
\end{array}$ & $\begin{array}{l}- \\
- \\
\end{array}$ & $\begin{array}{c}P, t p, f \\
0.52 \\
\end{array}$ \\
\hline Jelenia Góra & $\begin{array}{l}\text { P. I. } \\
R_{p}^{2}\end{array}$ & $\begin{array}{c}T \\
0.29\end{array}$ & $\begin{array}{c}P, T, v \\
0.28\end{array}$ & $\begin{array}{c}T \\
0.20\end{array}$ & $\begin{array}{l}\# \\
\# \#\end{array}$ & \# & $\begin{array}{l}\# \\
\# \#\end{array}$ & $\begin{array}{l}T, v \\
0.39\end{array}$ \\
\hline
\end{tabular}

Explanations: P. I. - statistically significant parameter, $R_{p}{ }^{2}$ - corrected determination coefficient, \# lack of statistically significant parameters, \#- value not defined.

Source: own study. 
Aside of wind speed, the variation of imission of the air pollutants analyzed was also affected by other meteorological parameters, determined with the stepwise procedure of regression analysis (Tab. 5). The variation of the determination coefficients $R_{p}^{2}$ that determine the relations between concentrations and selected group of meteorological elements was within a very broad range (from 0.10 to 0.76 ). This depended on the month analyzed and monitoring station. In all the months of the winter periods of the 2005/2006 2008/2009 the meteorological conditions affected most the concentrations of $\mathrm{O}_{3}, \mathrm{CO}, \mathrm{NO}_{2}$ and $\mathrm{SO}_{2}$ (wherever they were measured). In April there were no significant relations for $\mathrm{NO}, \mathrm{NO}_{2}$ and $\mathrm{NO}_{\mathrm{x}}$ at Wrocław-Swojec monitoring station, and for $\mathrm{SO}_{2}$ and PM10 at Jelenia Góra. The lowest coefficients $R_{p}{ }^{2}$ were observed for NO in all winter-period months.

In December, January and April the concentrations of pollutants were described by two or three meteorological elements, the most often indicated by the step-wise procedure was wind speed, then total solar radiation, air temperature and relative humidity. Only in April atmospheric precipitation significantly influenced the concentration of air pollutants. In February and March, in most of the cases, statistically significant effect of weather on imission was limited to single meteorological elements, from which, independently from station, wind speed was the most frequent typed parameter, then air temperature, less often total solar radiation and air relative humidity.

Comparable influence of air temperature and wind speed on PM10 was confirmed by the results obtained for Poland in a point scale with the application of similar methods of analysis [CZARNECKA, NIDZGORSKA-LENCEWICZ 2011; NIDZGORSKA-LENCEWICZ, CZARNECKA 2014]. On the contrary, analysis performed by GIRI et al. [2008] showed that the atmospheric pressure, wind speed and air relative humidity were significant factors influencing PM10 concentration. Similarly air temperature was found as the main factor influencing $\mathrm{SO}_{2}$ concentration during most of winter month in other countries i.e. by BANERJEE et al. [2011], LUVSAN et al. [2012], ÇELIK and KADI [2007]. Analysis indicated that wind speed was the meteorological element correlated most with the concentrations of $\mathrm{CO}, \mathrm{O}_{3}$ and $\mathrm{NO}_{\mathrm{x}}$ [KATSOULIS 1996]. Air relative humidity and total solar radiation were the factors also strongly influencing $\mathrm{O}_{3}$ concentration, as presented in the paper. Obtained results showed that air pollution is an area very sensitive to individual meteorological parameters.

\section{CONCLUSIONS}

1. High variability of analyzed values of pollutant concentrations was observed for selected air quality monitoring stations.

2. Maximum concentration of $\mathrm{NO}, \mathrm{NO}_{2}, \mathrm{NO}_{\mathrm{X}}$ and $\mathrm{SO}_{2}$ occurred in the coldest months during winter season (January, February and December). That is prob- ably the result of strong influence of "low emission" on air quality. Such conclusion is not possible for PM10 due to the insufficient database for that parameter.

3. Among the meteorological factors assessed the concentration of gaseous and dust pollutants was mainly affected by wind speed, whose increase resulted in decreased concentration of air pollution.

4. Total solar radiation, air relative humidity and temperature had much less impact on air quality. Such a tendency was observed for all analyzed monitoring stations.

5. During winter season, (except April in Dzierżoniów station) no significant correlations were obtained for the relation between atmospheric precipitation and analyzed pollutants.

6 . In the case of a larger number of variables describing the pollution in the atmosphere, in all the analyzed winter seasons the most common set of meteorological elements were wind speed and air temperature.

\section{REFERENCES}

AKPINAR E.K., ÖZTOP H.F. 2008. Evaluation of relationship between meteorological parameters and air pollutant concentrations during winter season in Elaziğ, Turkey. Environmental Monitoring and Assessment. Vol. 146 p. 211224.

AKPINAR E.K., AKPINAR S., ÖZToP H.F. 2009. Statistical analysis of meteorological factors and air pollution at winter months in Elaziğ, Turkey. Journal of Urban and Environmental Engineering. Vol. 3(1) p. 7-16.

BanerJee T., Singh S.B., Srivastava R.K. 2011. Development and performance evaluation of statistical models correlating air pollutants and meteorological variables at Pantnagar, India. Atmospheric Research. Vol. 99 p. 505517.

Bridgman H.A., Davies T.D., Jickells T., Hunova I., Tovey K., Bridges K., SURAPIPITH V. 2002. Air pollution in the Krusne Hory region, Czech Republic during the 1990s. Atmospheric Environment. Vol. 36 p. 33753389.

Camalier L., Cox W., Dolwick P. 2007. The effects of meteorology on ozone in urban areas and their use in assessing ozone trends. Atmosphere Environment. Vol. 41 p. $7127-7137$

ÇELIK M.B., KADI İ. 2007. The relation between meteorological factors and pollutants concentrations in Karabük City. G.U. Journal of Science. Vol. 20(4) p. 87-95.

Cuhadaroglu B., Demirci E. 1997. Influence of some meteorological factors on air pollution in Trabzon city. Energy and Building. Vol. 25 p. 179-184.

CZARNECKA M., KALBARCZYK R., KALBARCZYK E. 2007. Variability in particulate master concentration versus precipitation in Pomerania region. Polish Journal of Natural Science. Vol. 22(4) p. 645-659.

CZARNECKA M., KALBARCZYK R. 2008. Warunki meteorologiczne kształtujące zmienność stężenia pyłu zawieszonego na Pomorzu [Weather conditions determining variability of suspended particulate matter concentration in Pomerania]. Acta Agrophysica. Vol. 11(2) p. 357-368.

CZARNECKA M., NiDZGORSKA-LENCEWICZ J. 2008. Warunki meteorologiczne kształtujące jakość powietrza w styczniu 
i w lipcu 2006 roku w Szczecinie [Meteorological conditions determining the quality of air in Szczecin in January and in July 2006]. Acta Agrophysica. Vol. 12(1) p. 5572.

CZARNECKA M., NidZGORSKA-LENCEWICZ J. 2011. Impact of weather conditions on Winter and summer air quality. International Agrophysics. Vol. 25(1) p. 7-12.

Giri D., Krishna Murthy V., AdHIKARY P.R. 2008. The influence of meteorological conditions on $\mathrm{PM}_{10}$ concentrations in Kathmandu Valley. International Journal of Environmental Resources. Vol. 2(1) p. 49-60.

GLIŃSKi J., STĘPNIEWSKA Z., STĘPNIEWSKI W., OSTROWSKI J., SZMAGARA A. 2000. A contribution to the assessment of potential denitrification in arable minerals soils of Poland. Journal of Water and land Development. No.4 p.175-183.

KATSOULIS B.D. 1996. The relationship between synoptic, mesoscale and microscale meteorological parameters during poor air quality events in Athens, Greece. The Science of the Total Environment. No 181 p. 13-24.

Luvsan M.E., ShiE R.H., Purevdoru T., BADARCh L., BALDORJ B., CHAN C.C. 2012. The influence of emission sources and meteorological conditions on $\mathrm{SO}_{2}$ pollution in Mongolia. Atmospheric Environment. Vol. 61 p. 542549.

NiDZGORSKA-LENCEWICZ J., CZARNECKA M. 2014. Winter weather conditions vs. air quality in Tricity, Poland. The- oretical and Applied Climatology. DOI 10.1007/ s00704014-1129-8.

Pearce J.L., Beringer J., Nicholls N., Hyndman R.J., TAPPER N.J. 2011. Quantifying the influence of local meteorology on air quality using generalized additive models. Atmospheric Environment. Vol. 45 p. 1328-1336.

SapeK A., NAwalny P., Barszczewski J. 2003. Ładunek składników nawozowych wnoszonych z opadem mokrym na powierzchnię ziemi w Falentach w latach 1995-2001 [Nutrient load with wet precipitation on the soil surface in Falenty during 1995-2001]. Woda-Środowisko-Obszary Wiejskie. T. 3. Z. specj. (6) p. 67-77.

STANISZ A. 2007. Przystępny kurs statystyki z zastosowaniem STATISTICA PL na przykładach z medycyny. T. 2. Modele liniowe i nieliniowe [Approachable statistics course using STATISTICA PL examples from medicine. T. 2. Linear and nonlinear models]. Kraków. Wydaw. StatSoft Polska. ISBN 83-912346-4-9 pp. 408.

TuRALIOĞLU F.S., NuHOĞLU A., BAYRAKTAR H. 2005. Impacts of some meteorological parameters on $\mathrm{SO}_{2}$ and TSP concentrations in Erzurum, Turkey. Chemosphere. Vol. 59 p. $1633-1642$.

Twardy S., KopaCz M., JanOtA D. 2005. Chemical loads carried by atmospheric precipitation and surface waters in the Małe Pieniny. Journal of Water and Land Development. No. 9 p. $147-160$.

\title{
Andrzej ŻYROMSKI, Małgorzata BINIAK-PIERÓG, Ewa BURSZTA-ADAMIAK, Zenon ZAMIAR
}

\author{
Ocena zależności między wielkością stężenia zanieczyszczeń powietrza a elementami meteorologicznymi w \\ okresie zimowym
}

\section{STRESZCZENIE}

Słowa kluczowe: elementy meteorologiczne, okres zimowy, regresja krokowa, temperatura, zanieczyszczenie powietrza

W pracy przedstawiono wyniki badań mające na celu ocenę związków między stężeniem zanieczyszczeń powietrza a elementami meteorologicznymi.

Analizą objęto średniodobowe wartości imisji zanieczyszczeń gazowych i pyłowych oraz wybrane elementy meteorologiczne, tj. prędkość wiatru, temperaturę, wysokość opadu atmosferycznego, wilgotność względną oraz promieniowanie całkowite na czterech stacjach pomiarowych zlokalizowanych na terenie województwa dolnośląskiego w okresie zimowym (listopad-kwiecień, zgodnie z klasyfikacją roku hydrologicznego) dla lat 20052009. Dane te pochodziły z badań z automatycznych sieci monitoringu zanieczyszczeń powietrza prowadzonych w ramach Państwowego Monitoringu Środowiska. Wpływ elementów meteorologicznych na stężenie analizowanych zanieczyszczeń określono z zastosowaniem analizy korelacji oraz regresji krokowej na poziomie istotności $\alpha<0,05$.

Występowanie maksymalnych wartości stężenia $\mathrm{NO}, \mathrm{NO}_{2}, \mathrm{NO}_{\mathrm{X}}$ oraz $\mathrm{SO}_{2}$ w najchłodniejszych miesiącach okresu zimowego (styczeń, luty i grudzień) potwierdziło duży wpływ „małej emisji” na jakość powietrza. Elementami meteorologicznymi najczęściej wskazywanymi w opisach zależności i za pomocą procedury regresji krokowej były prędkość wiatru, następnie temperatura powietrza i rzadziej wilgotność względna powietrza, temperatura powietrza, promieniowanie całkowite oraz wilgotność powietrza. W przypadku występowania większej ilości zmiennych opisujących zanieczyszczenia w atmosferze, we wszystkich analizowanych półroczach zimowych najczęściej występującym układem elementów meteorologicznych była prędkość wiatru i temperatura powietrza. 